\title{
The Null Space Pursuit Algorithm based on an Arbitrary Even-Order Differential Operator
}

\author{
W. W. Xiao \& Y. X. Guo \\ North China University of Technology, Beijing, China
}

\begin{abstract}
The null space pursuit (NSP) algorithm based on the differential operator is a method of signal separation. In this paper, we propose an arbitrary even-order differential operator and estimate the parameters of the differential operator by solving an optimization problem. Moreover, we use experimental simulation to test the effectiveness of the proposed method.
\end{abstract}

KEYWORD: Null space pursuit algorithm; Signal separation; Differential operator

\section{INTRODUCTION}

Recently, the single-channel signal separation problem, which involves decomposing a signal into its coherent subcomponents, has attracted much attention because it affects lots of applications. The methods used to separate signals vary because different subcomponents are used to construct the signals. For example, in the empirical mode decomposition (EMD) approach[1,2] , an oscillatory signal is decomposed into a sum of intrinsic mode functions (IMFs); and in the matching pursuit (MP) approach[3], a signal is decomposed into a sum of time-frequency atoms. In all methods of signal separation, the null space pursuit (NSP) algorithm based on a differential operator is of particular interest to us.

In 2008 [4], Silong Peng and Wen-Liang Hwang proposed the NSP algorithm based on a differential operator, and they further improved it in 2010 [5]. This method makes use of an adaptive operator to decompose a signal into a sum of simple signals, and these simple signals belong to the null space in the above. The important procedures of the method include estimating the adaptive operator $T_{s}$ from the input signal $S$ and decomposing it into $R$ and $U$, where $R$ is the residual signal and $U$ is extracted signal, which $T_{s}(U)=0$. In [5], they proposed the following second-order differential operator: $T_{s}=\frac{d^{2}}{d t^{2}}+\omega^{2}(t)$, which can annihilate the FM signal $\cos (\phi(t))$, where $\phi(t)$ is a local linear function.
The NSP algorithm based on a differential operator has attracted wide attention because of its adaptability. In 2011, Xiyuan $\mathrm{Hu}$ [6] improved the null space pursuit algorithm and then further expanded the scope of signal that could be decomposed. He proposed the following second-order differential operator: $T_{s}=\frac{d^{2}}{d t^{2}}+P(t) \frac{d}{d t}+Q(t)$. It can annihilate an AM-FM signal.

In this paper, we improve the algorithm in [5] to use an arbitrary even-order differential operator. It can annihilate the following signal: $\cos \left(\phi_{1}(t)\right)+\cos \left(\phi_{2}(t)\right)+\cdots+\cos \left(\phi_{m}(t)\right)$. What's more, the algorithm proposed by us expands the scope of signal that could be decomposed.

\section{NULL SPACE PURSUIT ALGORITHM BASED ON ARBITRARY EVEN-ORDER DIFFERENTIAL OPERATOR}

In this paper, we propose the following arbitrary even-order differential operator:

$T_{s}=\frac{d^{2 m}}{d t^{2 m}}+\alpha_{m-1}(t) \frac{d^{2(m-1)}}{d t^{2(m-1)}}+\cdots+\alpha_{1}(t) \frac{d^{2}}{d t^{2}}+\alpha_{0}(t)$.

This algorithm can estimate the orders of the above operator and the values of parameters $\alpha_{0}, \alpha_{1}, \cdots, \alpha_{m-1}$.

In a discrete case, the form of the operator is denoted as: 
$T_{s}=D_{2 m}+P_{\alpha_{m-1}} D_{2(m-1)}+\cdots+P_{\alpha_{1}} D_{2}+P_{\alpha_{0}}$,

Where $P_{\alpha_{i}}$ is a diagonal matrix whose diagonal elements are equal to $\alpha_{i}$, where $i=0,1, \cdots, m-1$, and $D_{n}(n=2 k, k=1,2,3, \cdots) \quad$ is the matrix of the $n$-order difference.

Then, the following optimization method is used to estimate the values of parameters $\alpha_{0}, \alpha_{1}, \cdots, \alpha_{m-1}$ and the signal $R$ that minimizes the problem:

$$
\begin{array}{r}
\min _{\alpha_{0}, \cdots, \alpha_{m-1}, R, \lambda_{1}, \gamma, \lambda_{2}}\left\{T_{s}(S-R) \|^{2}+\lambda_{1}\left(\|R\|^{2}+\gamma\|S-R\|^{2}\right)\right. \\
\left.+\lambda_{2}\left(\left\|D_{2} \alpha_{m-1}\right\|^{2}+\cdots+\left\|D_{2} \alpha_{0}\right\|^{2}\right)\right\}
\end{array}
$$

Where $S$ is the input signal, $R$ is the residual signal, $\lambda_{1}, \lambda_{2}$ are Lagrange parameters and $\gamma$ is the leakage parameter. Let

$$
\begin{aligned}
F\left(\alpha_{0}, \cdots, \alpha_{m-1}, R\right) & =\left\|T_{s}(S-R)\right\|^{2} \\
& +\lambda_{1}\left(\|R\|^{2}+\gamma\|S-R\|^{2}\right) \\
& +\lambda_{2}\left(\left\|D_{2} \alpha_{m-1}\right\|^{2}+\cdots+\left\|D_{2} \alpha_{0}\right\|^{2}\right)
\end{aligned} .
$$

For convenience, we let $\phi$ be the column vector as follows: $\phi=\left[\alpha_{m-1}^{T}, \alpha_{m-2}^{T}, \cdots, \alpha_{1}^{T}, \alpha_{0}^{T}\right]^{T}$. Then becomes

$$
\begin{aligned}
F(\phi, R) & =\left\|\left(D_{2 m}+B_{\phi} M_{1}\right)(S-R)\right\|^{2} \\
& +\lambda_{1}\left(\|R\|^{2}+\gamma\|S-R\|^{2}\right)+\lambda_{2}\left(\left\|M_{2} \phi\right\|^{2}\right)
\end{aligned},
$$

Where

$$
B_{\phi}=\left[P_{\alpha_{m-1}}, P_{\alpha_{m-2}}, \cdots, P_{\alpha_{1}}, P_{\alpha_{0}}\right], M_{2}=\left(\begin{array}{ccc}
D_{2} & & \\
& \ddots & \\
& & D_{2}
\end{array}\right)
$$

and $M_{1}=\left[D_{2(m-1)}^{T}, D_{2(m-2)}^{T}, \cdots, D_{2}^{T}, E^{T}\right]^{T}$,

in which $E$ is the identity matrix.

For convenience, we rewrite the first item of (3) as the following:

$$
\left(D_{2 m}+B_{\phi} M_{1}\right)(S-R)=D_{2 m}(S-R)+B_{\phi} M_{1}(S-R)
$$

$$
\begin{aligned}
& =D_{2 m}(S-R)+B_{\phi}\left(\begin{array}{c}
D_{2(m-1)}(S-R) \\
\vdots \\
D_{2}(S-R) \\
E(S-R)
\end{array}\right) \\
& =D_{2 m}(S-R)+P_{D_{2(m-1)}(S-R)} \alpha_{m-1}+\cdots+P_{(S-R)} \alpha_{0} \\
& =D_{2 m}(S-R)+A \phi
\end{aligned}
$$

Where $A=\left[P_{D_{2(m-1)}(S-R)}, \cdots, P_{D_{2}(S-R)}, P_{(S-R)}\right]$. Then (3) becomes

$$
\begin{gathered}
F(\phi, R)=\left\|D_{2 m}(S-R)+A \phi\right\|^{2}+\lambda_{1}\left(\|R\|^{2}+\gamma\|S-R\|^{2}\right) . \\
+\lambda_{2}\left(\left\|M_{2} \phi\right\|^{2}\right)
\end{gathered}
$$

We assume $\frac{\partial F}{\partial \phi}=2 A^{T}\left(D_{2 m}(S-R)+A \phi\right)+2 \lambda_{2} M_{2}^{T} M_{2} \phi=0$.

Then

$\hat{\phi}=-\left(A^{T} A+\lambda_{2} M_{2}^{T} M_{2}\right)^{-1} A^{T} D_{2 m}(S-R)$.

Similarly, we use $\left.\frac{\partial F}{\partial R}\right|_{\phi=\hat{\phi}}=0$ to estimate $\hat{R}$ and obtain

$$
\begin{aligned}
\hat{R} & =\left(T_{s}^{T} T_{s}+\lambda_{1}(1+\gamma) E\right)^{-1}\left(T_{s}^{T} T_{s} S+\lambda_{1} \gamma S\right), \\
& =M\left(\hat{\lambda}_{1}, \hat{\gamma}\right)\left(T_{s}^{T} T_{s} S+\lambda_{1} \gamma S\right)
\end{aligned}
$$

Where

$T_{s}=D_{2 m}+P_{\alpha_{m-1}} D_{2(m-1)}+\cdots+P_{\alpha_{2}} D_{4}+P_{\alpha_{1}} D_{2}+P_{\alpha_{0}}$ $M\left(\widehat{\lambda}_{1}, \hat{\gamma}\right)=\left(T_{s}^{T} T_{s}+\lambda_{1}(1+\gamma) E\right)^{-1}$.

For the NSP algorithm, parameters $\lambda_{1}$ and $\gamma$ can be calculated as follows:

$$
\begin{aligned}
& \frac{1}{1+\hat{\gamma}} \frac{S^{T} M\left(\lambda_{1}, \hat{\gamma}\right)^{T} S}{S^{T} M\left(\lambda_{1}, \hat{\gamma}\right)^{T} M\left(\lambda_{1}, \hat{\gamma}\right) S}=\lambda_{1} \\
& \frac{(S-\hat{R})^{T} S}{\|S-\hat{R}\|^{2}}-1=\gamma
\end{aligned}
$$

However, the optimal value of $\lambda_{2}$ is failed to estimate by the above procedure. In practice, we find the optimal solution of (2) is insensitive to the value of $\lambda_{2}$. Thus, the value can be fixed.

\section{NULL SPACE PURSUIT ALGORITHM}

We assume that the input signal is $s(t)$, and the value of $\lambda_{2}$ is given.

1) Input: the initial values of $\lambda_{1}^{0}$ and $\gamma^{0}$, the stopping threshold $\epsilon$; Let $k=1$;

2) Let $j=0, \hat{r}_{j}=0, \lambda_{1}^{j}=\lambda_{1}^{0}, \gamma^{j}=\gamma^{0}$;

3) Compute $\phi_{j}$ according to (6) by signal $\hat{r}_{j}$; Then, obtain the values of $\hat{\alpha}_{0}, \hat{\alpha}_{1}, \cdots, \hat{\alpha}_{m-1}$;

4) Compute $\lambda_{1}^{j+1}$ according to (8) by $\phi_{j}, \hat{r}_{j}, \lambda_{1}^{j}, \gamma^{j}$;

5) Compute $\hat{r}_{j+1}$ according to (7) by $\lambda_{1}^{j+1}, \phi_{j}, \gamma^{j}$;

6) Compute $\gamma^{j+1}$ according to (9) by $\hat{r}_{j+1}$, then set $j=j+1$;

7) If $\left\|\hat{r}_{j+1}-\hat{r}_{j}\right\|^{2}>\epsilon$, go to step 3 ); otherwise, go to the next step;

8) Output the extracted signal $\hat{u}=\left(1+\gamma^{j+1}\right)\left(s-\hat{r}_{j}\right)$ and the residual signal $\hat{r}=s-\hat{u}$; 
9) If $\|\hat{r}\|^{2}>\epsilon\|s\|^{2}$, then set $k=k+1$ and go to step 2); otherwise, stop the program.

\section{EXPERIMENT RESULT AND ANALYSIS}

In this section, we present several examples to demonstrate the results achieved by our algorithm.

In the first example, the input signal is the signal $\cos (t)+\cos (3 t)$ in additive Gaussian random noise, as shown in Figure 1. By executing a Matlab program, we obtain the PSNR of input signal as $25.9819 \mathrm{~dB}$, and the PSNR of extracted signal as $37.4213 \mathrm{~dB}$.

Let $\gamma^{0}=1, \lambda_{1}=0.0001, \lambda_{2}=0.0001, \varepsilon=0.1$. This example shows that our algorithm can estimate well the orders of differential operator, and have a good effect to signal denoising.
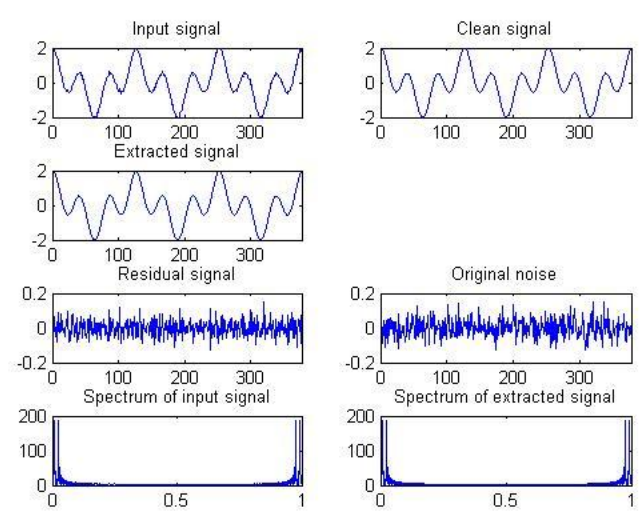

Figure 1. Signal denoising

The second example shows that our algorithm can remove the noise from a noisy chirp signal and decompose the extracted signal into its coherent subcomponents. First, we input the signal $\cos (t)+\cos (3 t)+\cos (7 t)$ in additive Gaussian random noise. Figure 2 shows that the extracted is closed to the clean signal. And it can estimate a sixth-order differential operator. By executing a Matlab program, we obtain the PSNR of input signal as $28.2468 \mathrm{~dB}$, and the PSNR of extracted signal as $31.8285 \mathrm{~dB}$. In this procedure, let $\gamma^{0}=1, \lambda_{1}=0.0001, \lambda_{2}=0.001, \varepsilon=0.1 \quad$.Then, we separate the extracted signal into three subcomponents. From the picture, the first component is closed to the signal $\cos (t)$, the second component is closed to the signal $\cos (3 t)$, and the residual signal is closed to the signal $\cos (7 t)$. The values of parameters for extraction of the first and second subcomponents are set at $\gamma^{0}=1, \lambda_{1}=0.0001$, $\lambda_{2}=0.0001, \varepsilon=0.01$ and $\gamma^{0}=1, \lambda_{1}=0.001, \lambda_{2}=0.001$ $\varepsilon=0.001$.

\section{SUMMARY}

In this paper, we propose an NSP algorithm based on an arbitrary even-order differential operator. It expands the scope of signal that could be decomposed comparing with the former one. In our future work, we will improve the order of differential operator and extend the method to images.
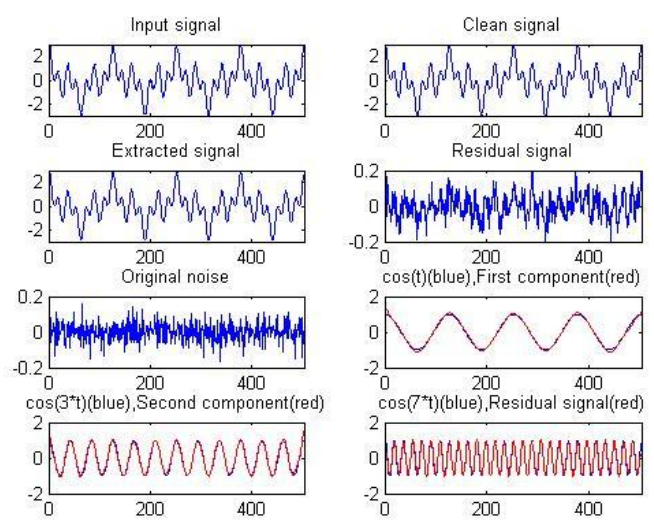

Figure 2. Signal denoising and signal separation

\section{ACKNOWLEDGMENT}

This work was supported by National Natural Science Foundation of China (11126140, 11201007); Beijing Talents Training Program (2011D005002000006); Science and Technology Development Plan Project of Beijing Education Commission (KM201210009013); Scientific Research Personnel Promotion Plan of North China University of Technology (BJRC201309).

\section{REFERENCES}

[1] Wu, Z. \& Huang, N.E. 2004. A study of the characteristics of white noise using the empirical mode decomposition method. Proceedings of the royal society of London (series A) 460: 1597-1611.

[2] Rilling, G. \& Flandrin, P. 2008. One or two frequencies? The empirical mode decomposition answers. IEEE Trans. Signal Process.56: 85-95.

[3] Mallat, S. \& Zhang, Z. 1993. Matching pursuits with time-frequency dictionaries. IEEE Trans. Signal Process41:3397-3415.

[4] Peng, S.L. \& Hwang, W.L. 2008. Adaptive signal decomposition based on local narrow band signals. IEEE Trans Signal Process.56: 2669-2676.

[5] Peng, S.L. \& Hwang, W.L. 2010. Null space pursuit: an operator-based approach to adaptive signal separation. IEEE Trans Signal Process58: 2475-2483.

[6] $\mathrm{Hu}, \mathrm{X} . \mathrm{Y}$. Adaptive signal and image separation and its application, Dissertation for the degree of doctor of philosophy. Insititute of Automation Chinese Academy of Sciences.Beijing. 